\title{
Three-dimensional modeling of human airway development using induced pluripotent stem cell-derived teratomas
}

\author{
Sansac $\mathrm{C}^{1 *}$, Belbachir $\mathrm{A}^{2 *}$, Ahmed E ${ }^{1}$, Ramirez JM${ }^{1}$, Fort $\mathrm{A}^{3}$, Péquignot $\mathrm{M}^{4}$, Serre $\mathrm{I}^{5}$, Penault-Llorca $\mathrm{F}^{6}$, Assou $\mathrm{S}^{1}$, Bourdin $\mathrm{B}^{3,7}$ and De Vos J,8 \\ ${ }^{1}$ IRMB, Univ Montpellier, INSERM, CHU Montpellier, Montpellier, F34000 France \\ ${ }^{2}$ Faculty of Medicine and Pharmacy, Cadi Ayyad University, 2390 Marrakech, Morocco \\ ${ }^{3}$ INSERM, U1046, Montpellier, F34000 France \\ ${ }^{4}$ Institute for Neurosciences of Montpellier, Montpellier, F34000 France \\ ${ }^{5}$ CHRU Montpellier, Department of Pathology, Montpellier, F34000 France \\ ${ }^{6}$ Department of Pathology, Centre Jean Perrin, EA 4677, Université d'Auvergne Clermont 1, F63000 Clermont-Ferrand, France \\ ${ }^{7}$ Department of Respiratory Diseases, Hôpital Arnaud de Villeneuve, Montpellier, F34000 France \\ ${ }^{8} \mathrm{CHU}$ Montpellier, Department of Cell and Tissue Engineering, Hospital Saint-Eloi, Montpellier, F 34000 France
}

\begin{abstract}
Human induced pluripotent stem cells (hiPSC) are obtained by reprogramming differentiated cells into a pluripotent state. As they can differentiate in vitro into numerous (if not all) human cell types, hiPSC are a promising source of cells for modeling normal and diseased organs/tissues. However, in classical cell culture systems, hiPSC differentiation is limited by the fact that only monolayers of cells or oligo-layered structures are produced, mainly because of the absence of vasculature. Conversely, hiPSC injection in immunosuppressed mice induces teratomas (i.e., three-dimensional structures that include tissues from the three germinal layers). Here, we thoroughly investigated the extent of airway development in hiPSC-derived teratomas. Teratomas were produced by injecting undifferentiated hiPSC subcutaneously in NOD.Cg-Prkdc scid $\mathrm{I} 2 \mathrm{rg}^{\mathrm{tm} 1 \mathrm{~W}_{\mathrm{j}} \mathrm{l}} / \mathrm{SzJ}$ mice that were sacrificed after seven to ten weeks. Teratomas were collected, fixed in $4 \%$ paraformaldehyde and embedded in paraffin. Their structure and composition were analyzed by immunohistochemistry using different antibodies. Within hiPSC-derived teratomas, typical endodermal tubes were formed that comprised patches of airway epithelium. These epithelia expressed CK7, TTF1/NKX2.1 and CC10, but not CD20 and CDX2 (markers of intestinal epithelial differentiation) that were expressed only in CC10-negative areas. Well differentiated mesenchymal structures, such as smooth muscle bundles, vessels and cartilage, formed underneath the airway epithelial patches, thus mimicking the normal airway wall. These results show that hiPSC-derived teratomas are a promising tool to study airway development and to model hereditary lung diseases.
\end{abstract}

\section{Introduction}

For obvious ethical issues, human development can be studied in utero only with non-invasive techniques, such as echography, or in vitro in cultured embryos, but only until the blastocyst stage. Human pluripotent stem cells (hPSC) can differentiate into any cell type of the body [1]. They are derived from the blastocyst inner cell mass (human embryonic stem cells, hESC), or by reprogramming differentiated cells into human induced pluripotent stem cells (hiPSC) through forced expression of reprogramming factors, such as OCT4, SOX2, c-MYC and KLF4 [2,3]. However, upon differentiation, cultured hPSC produces only monolayers of cells or oligo-layered structures, partly because of the absence of vasculature. By contrast, hPSC injection in immunosuppressed mice to form teratomas is a useful three-dimensional (3D) system that mimics more closely early human development. Indeed, teratomas can make use of the mouse's vasculature to form relatively large structures. This allows: (1) the formation of a $3 \mathrm{D}$ structure that reproduces physiological embryo development, including the complex interactions between different cell types, (2) cell/tissue differentiation during a long period (i.e., at least two months). Indeed, teratomas are made of many different tissues that originated from the three germ layers and that often show the microscopic morphology of the equivalent fetal human somatic tissues. However, the presence of lung tissue within teratomas, has only rarely been mentioned [4-6], although it is a major organ of endodermal origin. Here we investigated the extent of airway development in hiPSC-derived teratomas.

\section{Materials and methods}

\section{Cell culture}

Human foreskin fibroblasts (hFF1 cell line from ATCC) were cultured in DMEM supplemented with $10 \%$ fetal calf serum. The

*Correspondence to: John De Vos, Department of Cell and Tissue Engineering, Hôpital Saint-Eloi, 80 Avenue Augustin Fliche, 34000 Montpellier Cedex 5, France, E-mail: john.devos@inserm.fr

Arnaud Bourdin, Department of Respiratory Diseases, 371 Av Doyen Giraud, 34000 Montpellier Cedex 5, France, E-mail: a-bourdin@chu-montpellier.fr

Received: April 03, 2018; Accepted: April 24, 2018; Published: April 27, 2018 
hiPSC line M4C7 was cultured on irradiated human foreskin fibroblasts with KO-DMEM supplemented with 10\% KO-SR (Gibco, Life Technologies $\left.^{\circledR}\right)$. The hiPSC line iPCD02-30 was cultured in feederfree conditions on Matrigel (BD Biosciences) in $\mathrm{mTeSR}^{\mathrm{ma}} 1$ medium (StemCell Technologies), or on Geltrex matrix in E8 medium (Gibco, Life Technologies ${ }^{\circledR}$ ).

\section{hiPSC generation}

The two hiPSC lines M4C7 and iPCD02-30 were obtained by reprogramming human skin fibroblasts using lentiviral vectors that encode the open reading frames (ORFs) of human POU5F1, SOX2, NANOG and LIN28 [7], or the CytoTune-iPS 2.0 Sendai Reprogramming Kit, respectively. The hFF1 cell line (ATCC) was used to derive the M4C7 hiPSC line and skin fibroblasts from a donor after informed consent for the iPCD02-30 line. FACS, immunofluorescence, RT-qPCR or phosphatase alkaline activity analyses demonstrated that both cell lines had the typical hiPSC expression profile (Supplemental Fig S1 and S2). After injection in immunocompromised mice, they produced teratomas that included tissues from all three germ layers [8].

\section{Teratoma formation}

Undifferentiated M4C7 and iPCD02-30 cells were dissociated with Trypsin or Versen, and then $1 \times 10^{7}$ cells were mixed with Matrigel (BFD Biosciences) and injected subcutaneously in anesthetized NOD.CgPrkdc $c^{\text {scid }} \mathrm{Il} 2 \mathrm{rg}^{\mathrm{tm} 1 \mathrm{wj}_{\mathrm{jl}} / \mathrm{SzJ}}$ (NSG) mice [9]. Mice were sacrificed after seven to ten weeks. Teratomas were collected, fixed in $4 \%$ paraformaldehyde and embedded in paraffin. The teratomas 13F0351, 13F0353, 13F0355 and 13F0358 were derived from the M4C7 hiPSC line, and the teratomas 032, 103, 104, 105 and 106 from the iPCD02-30 line.

\section{Immunohistochemistry}

Paraffin-embedded teratoma sections were deparaffinized with xylene and rehydrated through alcohol gradient (100\% ethanol, 95\% ethanol, $70 \%$ ethanol, $50 \%$ ethanol). After heat-induced antigen retrieval in Tris-EDTA buffer $(100 \mathrm{mM}$ Tris, $1 \mathrm{mM}$ EDTA and Tween20, $\mathrm{pH}=9.0$ ), sections were blocked and incubated with primary antibodies against NKX2.1/TTF1 (Dako M3575), CC10 (BioVendor RD181022220), CK20 (Abcam ab15205), CK7 (Dako M7018 clone OV-TL 12/30), CDX2 (Biogenex MU392A-UC clone cdx288), Smooth muscle actin (Dako M0851 clone 1A4), Ki67 (Dako M7240 clone MIB-1), Vimentin (Santa Cruz SC7557), epithelial membrane antigen (EMA) (Novocastra NCL-MUC1/EMA clone Ma695), PGP9.5 (Abcam ab8189), Tubulin IV (Sigma-Aldrich ONS.1A6) and Muc5AC (Abcam $45 \mathrm{M} 1$ ) for $1 \mathrm{hr}$, and Aquaporin 5 (Abcam ab92320), HuNu (Merk MAB1281 Clone 235-1), overnight. Immunohistochemical staining was visualized using the avidin-biotin system (Dako, LSAB2 System-HRP) in combination with 3-3'-diaminobenzidine (DAB).

\section{Results}

\section{Teratomas have many tubular structures surrounded by mesenchymal tissue}

All hiPSC-derived teratomas displayed tubular structures (Figure 1A) composed of cuboidal or pseudostratified columnar epithelium, as indicated by the tightly packed cells that formed continuous sheets. The epithelium organization and the frequent presence of goblet cells indicated that these structures were of endodermal origin. The number of tubes varied from 3 to 59 per section, depending on the teratoma and the position of the section within the teratoma. These tubes seemed to be randomly associated with other tissue types, such as smooth muscle bundles (arrows) and vessels (arrowheads) (Figure 1A), or cartilage (arrows), which is an important component of the upper respiratory tract (Figure 1B). Cells in the endodermal tubes were mostly Ki67positive, a marker of cell proliferation, thus suggesting immature, developing structures (Figure 1C). Similarly, immature neural tissue, consisting of neural rosettes (arrows), was also frequently observed (Figure 1D). These epithelia developed on a basement membrane sheet that was underneath a vimentin-positive connective tissue layer (arrows in Figure 2). The interface between the epithelium and the subjacent mesenchymal tissue was very well delimited, indicating a highly organized developing tissue. Moreover, staining with an anti-smooth muscle actin antibody showed that smooth muscle cells surrounded the endodermal tubes, as typically observed in the airway and digestive tracts (arrows in Figure 2D).

\section{Opposing expression patterns of airway and digestive markers}

As endoderm gives rise to the epithelia that line the digestive and respiratory tracts, we studied the expression of the intermediate filament cytokeratin-7 (CK7; a cytoplasmic marker expressed in various epithelia, such as simple ductal epithelium, mesothelium and pseudostratified epithelium, but not in intestinal epithelium) [10], cytokeratin-20 (CK20; a cytoplasmic marker of the gastrointestinal tract) [11], thyroid transcription factor-1 (TTF1/NKX2.1; involved in lung, thyroid and forebrain specification) and CDX2 (a nuclear homeobox transcription factor expressed from the proximal duodenum to the distal rectum, midgut and hindgut, from week 8 of gestation and in postnatal life) [12]. CDX2 and CK20 are considered good markers of intestinal origin of metastatic adenocarcinoma, and CK7 of pulmonary origin in TTF1-positive/CK20-negative tumor samples [12-13]. In four M4C7-derived teratomas, most tubes expressed at least one or both cytokeratins $(74.0 \%)$ as well as TTF1 or/and CDX2 (75.6\%) (Table 1). Specifically, 41.8\% were CK7-positive and 22\% were TTF1-positive, while $47.2 \%$ were CK20positive and $70.7 \%$ CDX2-positive. CK7 often overlapped with
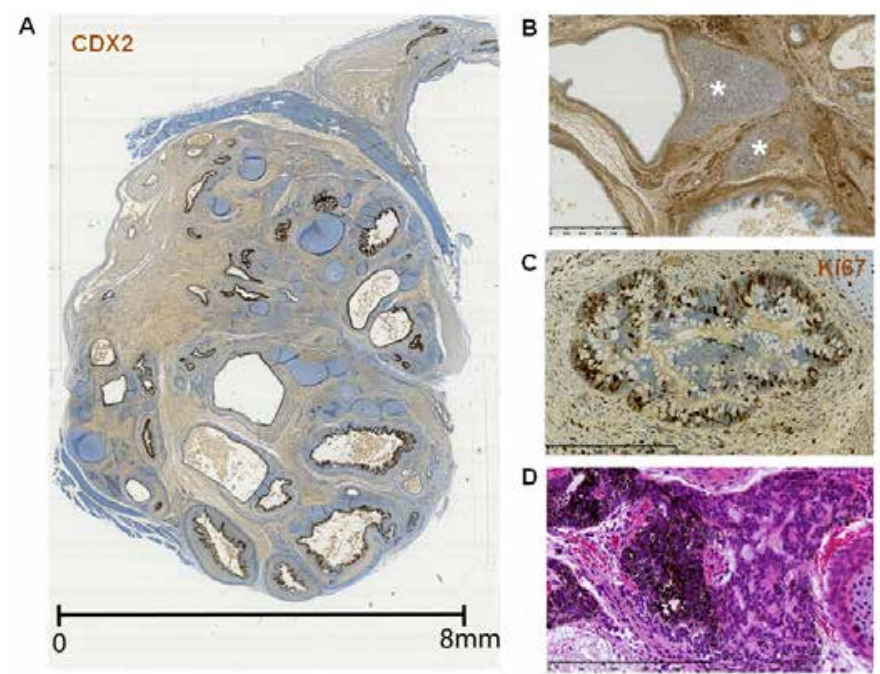

Figure 1. General organization of endodermal tubes in hiPSC-derived teratomas. All sections were counterstained with hematoxylin, eosin and saffron stain (HES). Antibody binding was visualized using the avidin-biotin system in combination with 3-3'-diaminobenzidine. (A) Whole section of the teratoma 13F0358 stained with an antiCDX2 antibody. (B) Proximity of cartilage (asterisks) with an endodermal tube. Teratoma 13F0358. (C) An endodermal tube stained with an anti-Ki67 antibody showing intense cell proliferation. Teratoma 13F0358. (D) Neural fetal tubes. Teratoma 104. 
Table 1. Number (and percentage) of tubular structures that express CK7 or/and CK20 (in all four M4C7-derived teratomas), or TTF1/NKX2.1 and/or CDX2 (only in teratomas 13F0351 and 13F0353). Tubular structures were counted in one representative section of each teratoma. The percentages indicate the fraction of tubes that express a specific protein relative to all the tubes in that section.

\begin{tabular}{|c|c|c|c|c|c|}
\hline $\begin{array}{c}\text { Expression } \\
\text { pattern }\end{array}$ & $13 F 0351$ & $13 F 0353$ & $13 F 0355$ & $13 F 0358$ & TOTAL \\
\hline $\begin{array}{l}\text { CK7+1 } \\
\text { CK20- }\end{array}$ & $\begin{array}{c}1 \\
(33.3 \%)\end{array}$ & $\begin{array}{c}15 \\
(75.0 \%)\end{array}$ & $\begin{array}{c}7 \\
(15.6 \%)\end{array}$ & $\begin{array}{c}11 \\
(18.6 \%)\end{array}$ & $\begin{array}{c}34 \\
(26.8 \%)\end{array}$ \\
\hline $\begin{array}{l}\text { CK7-/ } \\
\text { CK20+ }\end{array}$ & $\begin{array}{c}1 \\
(33.3 \%)\end{array}$ & $\begin{array}{c}1 \\
(5.0 \%)\end{array}$ & $\begin{array}{c}20 \\
(44.4 \%)\end{array}$ & $\begin{array}{c}19 \\
(32.2 \%)\end{array}$ & $\begin{array}{c}41 \\
(32.2 \%)\end{array}$ \\
\hline $\begin{array}{l}\text { CK7+/ } \\
\text { CK20+ }\end{array}$ & $\begin{array}{c}0 \\
(0.0 \%)\end{array}$ & $\begin{array}{c}3 \\
(15.0 \%)\end{array}$ & $\begin{array}{c}6 \\
(13.3 \%)\end{array}$ & $\begin{array}{c}10 \\
(16.9 \%)\end{array}$ & $\begin{array}{c}19 \\
(15.0 \%)\end{array}$ \\
\hline CK7-/CK20- & $\begin{array}{c}1 \\
(33.3 \%)\end{array}$ & $\begin{array}{c}1 \\
(5.0 \%)\end{array}$ & $\begin{array}{c}12 \\
(26.7 \%)\end{array}$ & $\begin{array}{c}19 \\
(32.2 \%)\end{array}$ & $\begin{array}{c}33 \\
(26.0 \%)\end{array}$ \\
\hline Total tubes & 3 & 20 & 45 & 59 & 127 \\
\hline $\begin{array}{c}\text { NKX2.1+l } \\
\text { CDX2- }\end{array}$ & - & - & $\begin{array}{c}1 \\
(9.1 \%)\end{array}$ & $\begin{array}{c}1 \\
(3.3 \%)\end{array}$ & $\begin{array}{c}2 \\
(4.9 \%)\end{array}$ \\
\hline $\begin{array}{c}\text { NKX2.1-/ } \\
\text { CDX2+ }\end{array}$ & - & - & $\begin{array}{c}6 \\
(54.5 \%)\end{array}$ & $\begin{array}{c}16 \\
(53.3 \%)\end{array}$ & $\begin{array}{c}22 \\
(53.6 \%)\end{array}$ \\
\hline $\begin{array}{c}\text { NKX2.1+l } \\
\text { CDX2+ }\end{array}$ & - & - & $\begin{array}{c}1 \\
(9.1 \%)\end{array}$ & $\begin{array}{c}6 \\
(20.0 \%)\end{array}$ & $\begin{array}{c}7 \\
(17.1 \%)\end{array}$ \\
\hline $\begin{array}{l}\text { NKX2.1-/ } \\
\text { CDX2- }\end{array}$ & - & - & $\begin{array}{c}3 \\
(27.3 \%)\end{array}$ & $\begin{array}{c}7 \\
(23.3 \%)\end{array}$ & $\begin{array}{c}10 \\
(24.4 \%)\end{array}$ \\
\hline Total tubes & - & - & 11 & 30 & 41 \\
\hline
\end{tabular}
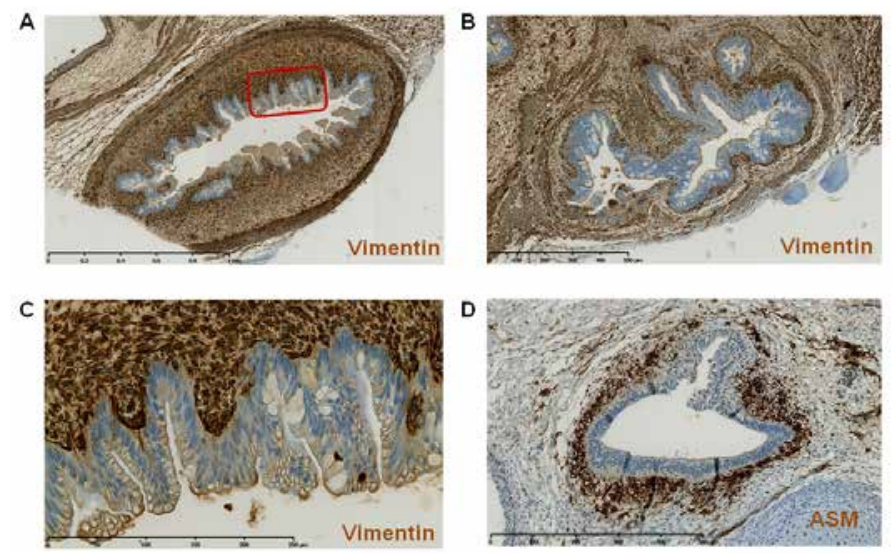

Figure 2. Interplay between the endodermal tube epithelium and the underlying mesenchymal tissue. (A-C) Teratoma 13F0358 sections stained with an anti-vimentin antibody. (C) Larger view of the boxed area in panel A. (D) Section from teratoma 13F0517 stained with an anti-actin smooth muscle (ASM) antibody to highlight smooth muscle.
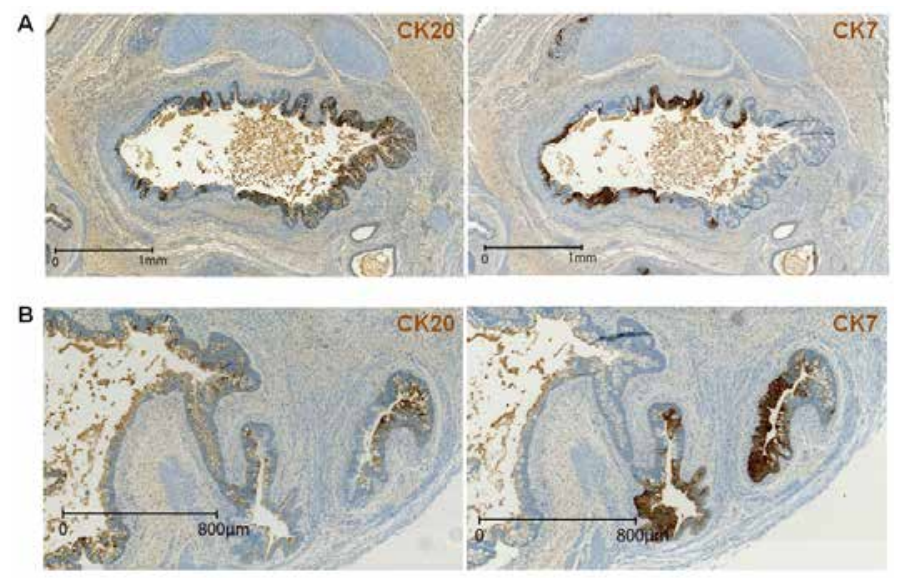

Figure 3. Opposing expression patterns of airway and digestive tract-specific cytokeratins. (A-B) Staining of consecutive sections of teratoma 13F0358 with the anti-CK20 and -CK7 antibodies shows the mutually exclusive expression patterns of CK7 (marker of airway tissue differentiation) and CK20 (marker of digestive tissue differentiation).
TTF1, and CK20 with CDX2, indicating the bronchial nature of the CK7/TTF1-positive epithelia and the digestive tract nature of CK20/ CDX2-positive epithelia (Figure 3 and 4). CK20 and CK7 (Figure 3) or CDX2 and TTF1 (Figure 4) were often concomitantly expressed in the same tube. However, CK7 or TTF1 never overlapped with CK20 or CDX2. For example, CK7 and CK20 expression were always mutually exclusive (Figure 3). Thus, teratomas included endoderm-derived epithelia that were simultaneously engaged in digestive tract and also in airway differentiation. The proportion of CK7-positive, CK20positive or CK20/CK7-positive tubes varied greatly within each and among teratomas, suggesting a chaotic determinism of the dominant differentiation direction in the endoderm tubes.

\section{Expression of mature airway markers in teratomas}

Analysis of the expression of SCGB1A1/CC10, a mature bronchial marker of club cells, showed that it was expressed specifically by epithelial cells in TTF1-positive, but never in TTF1-negative areas (Figure 5A). Mucin 1 (MUC1), also called epithelial membrane antigen (EMA), was expressed in CK7-positive tubes (Figure 5B). Furthermore, we used anti-CCSP, anti-tubulin IV and anti-MUC5AC antibodies to highlight the presence of club cells, ciliated cells, and goblet cells, respectively. Conversely, we did not detect expression of PGP9.5 (neuroendocrine cell marker) and of Aquaporin 5 (type I alveolar epithelial cell marker) (Figure 5C). These results indicate that patches of endoderm-derived epithelia display markers of maturing airway. Finally, we confirmed that mesenchyme and epithelial cells within teratomas were derived from the injected hiPSC by using a mouse anti$\mathrm{HuNu}$ antibody that marks human cells (Figure 5C).

\section{Discussion}

In this report, we show that hiPSC-derived teratomas can be used for 3D modelling of human bronchial epithelium differentiation as they include highly organized airway epithelial structures that are surrounded by vascularized mesenchyme and cartilage. As the hiPSC technology allows choosing the genetic background of the starting cell donor and specific genes can be knocked down by using the CRISP/ cas9 technology, it will be possible to mimic human embryonic airway development defects or the early steps of genetic diseases. Modeling normal or diseased human tissues is one of the nine applications of hiPSC [3]. Moreover, the teratoma system offers some advantages over common $2 \mathrm{D}$ differentiation protocols. The teratoma 3D microscopic architecture is closer to that of human tissues compared with $2 \mathrm{D}$ cell culture models. The complex and organized interconnection between the endodermal epithelium and the underlying vimentin-positive mesenchymal layer, of human origin (Figure 5C), is a good illustration of this feature. While 3D organoids can be used to generate differentiated tissues, including airway cells mixed with mesenchymal cells [14,15], the volume of tissue produced by teratomas is bigger, suggesting a better differentiation potential and an improved global architecture.

These observations suggest that teratomas recapitulate the dynamics of early human development, such as some aspects of gastrulation and the formation of endodermal tubes. Indeed, the intimate development of the mesenchymal layer underneath the endodermal tubes observed in teratomas mimics the normal airway development in which mesenchyme plays a crucial role [16]. Hence, hiPSC-derived teratomas might be a tool to study these essential early steps of airway development, for instance by using hiPSC in which specific growth factors or transcription factors have been genetically deleted.

Nonetheless, bona fide organogenesis is not taking place in teratomas and this might explain why the different tissues are scattered 
A
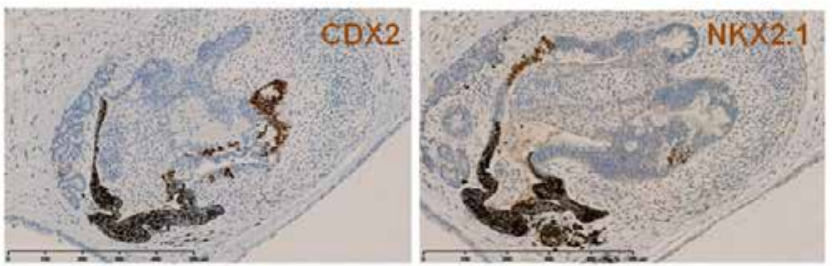

B
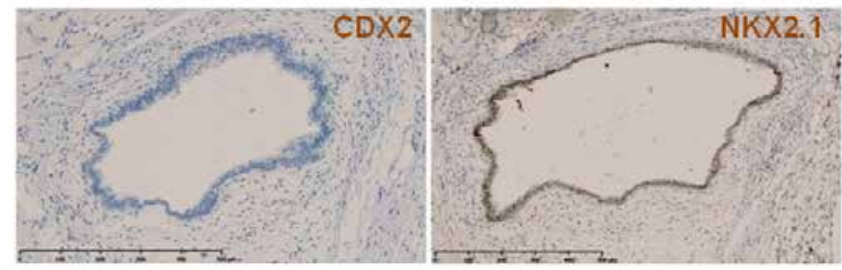

C

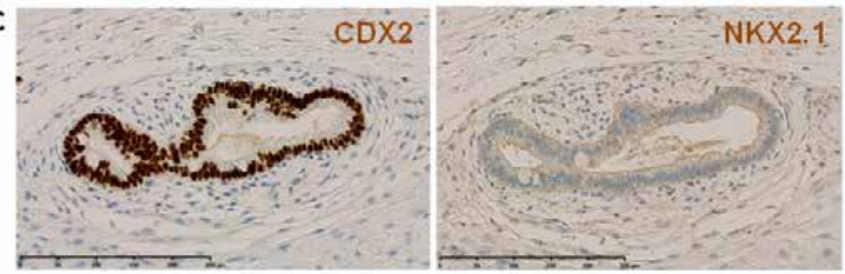

Figure 4. CDX2 and TTF1/NKX2.1 expression in endodermal tubes.

(A-C) Staining of consecutive sections with anti-CDX2 and -TTF1/NKX2.1 antibodies show the non-overlapping expression of TTF1/NKX2.1 (marker of airway tissue differentiation) and CDX2 (marker of digestive tissue differentiation) in the same endodermal tube (arrows) or the exclusive expression of one in other tubes (arrowheads). A. Teratoma 103; B and C: Teratoma 104.

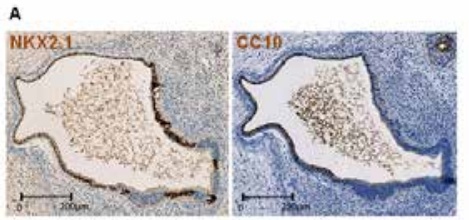

B
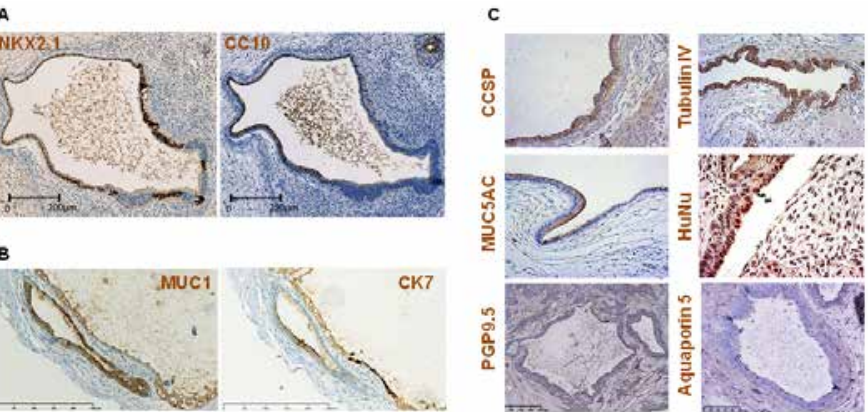

Figure 5. Late airway marker expression. (A) Two consecutive sections of teratoma 13F0517 stained with anti-TTF1/NKX2.1 and anti-CC10 (a mature bronchial marker of club cells) antibodies. CC10 is expressed only in TTF1/NKX2.1-positive areas. (B) Two consecutive sections of teratoma 13F0351 stained with anti-EMA and -CK7 antibodies show EMA expression in a CK7-positive endodermal tube. (C) Staining with anti-CCSP/ CC10 (teratoma 032), anti-tubulin IV (teratoma 106), anti-MUC5AC (teratoma 105), and anti-HuNu (teratoma 106) antibodies reveals the presence of club cells, ciliated cells, goblet cells, and human cells, respectively. Lack of signal for the anti-PGP9.5 (teratoma 103) and anti-Aquaporin 5 (teratoma 103) antibodies indicates the absence of neuroendocrine cells and of type I alveolar epithelial cells, respectively. An adult lung tissue section was used as positive control for Aquaporin 5 (data not shown).

without global organization [17]. For instance, in our teratomas, immature neural tubes can be close to endodermal tubes, something never observed during normal development. Moreover, a teratoma may contain many different endodermal tubes, while only one is generated during normal development. However, we cannot exclude that the different tubes observed in the same section are part of a unique tube. Moreover, in the same tube, TTF1/NKX2.1, a lung-specific transcription factor, could be expressed in close proximity to CDX2, a transcription factor expressed in the mid- and hindgut. Therefore, unless it is possible to manipulate and artificially direct differentiation to mimic the global organization observed in embryos, it will not be possible to use hiPSC- derived teratomas to model complex developmental processes, such as lung branching.

Teratoma formation is not easily amenable to experimental intervention to guide the differentiation process, differently from in vitro $2 \mathrm{D}$ or organoid culture models. Nonetheless, teratoma cell differentiation could be directed towards lung formation by at least two means. First, instead of injecting undifferentiated PSCs in immunodeficient mice, cells could be differentiated prior to injection. This strategy was used by Green et al. [18] to examine the developmental competence of human ESC-derived definitive endoderm cells in SCID mice. Similarly, Mou et al. [19] and Huang et al. [20] differentiated mouse or human hiPSC into TTF1/NKX2.1-positive cells that were injected subcutaneously in immunodeficient mice. These cells formed "tracheospheres" that contain terminally differentiated CC10-positive club cells, FOXJ1-positive ciliated cells and MUC5AC-positive goblet cells (for mouse cells) or P63-positive progenitor, basal cells (for human cells). Second, it may be possible to use soaked microbeads as a device to deliver specific growth factors or chemical inhibitors during teratoma formation. Such signals may reproduce some early events that take place during embryonic life and direct organogenesis towards a desired tissue type.

\section{Conclusion}

We have shown that human hiPSC-derived teratomas recapitulate the early steps of 3D airway development with the formation of epithelial tubes and the surrounding mesenchymal structures. These structures display marked morphological analogies with the airway structures found during early embryo development. Therefore, teratomas represent potential surrogate tools to study the mechanism of normal and abnormal early airway development.

\section{Compliance with ethical standards}

The derivation of the M4C7 and iPCD02-30 was approved by the Ethical committee "Comité de Protection des Personnes Sud Méditerranée IV" of Montpellier (Authorization number \#DC-20152473). All animal studies were conducted according to the European guidelines for animal welfare (2010/63/EU). Protocols were approved by the Languedoc Roussillon Institutional Animal Care and Use Committee (CEEA-LR-12157).

\section{Availability of data and materials}

The hiPSC lines used during the current study are available from the corresponding authors on reasonable request.

\section{Funding}

Supported by grants from the University Hospital of Montpellier (Appel d'offre interne, projet 9174), the association "Gueules Cassées" (Grant \#17-2015) and the association Vaincre la Mucoviscidose (Grant \#RIF20170502048).

\section{Conflicts of interest}

The authors declare no potential conflicts of interest.

\section{Acknowledgement}

We thank E. Andermarcher for critical reading of this manuscript.

\section{References}

1. Ramirez JM, Bai Q, Dijon-Grinand M, Assou S, Gerbal-Chaloin S, et al. (2010) Human pluripotent stem cells: from biology to cell therapy. World J Stem Cells 2: 24-33. [Crossref] 
2. Takahashi K, Tanabe K, Ohnuki M, Narita M, Ichisaka T, et al. (2007) Induction of pluripotent stem cells from adult human fibroblasts by defined factors. Cell 131: 861872. [Crossref]

3. De Vos J, Bouckenheimer J, Sansac C, Lemaitre JM, Assou S (2016) Human induced pluripotent stem cells: A disruptive innovation. Curr Res Transl Med 64: 91-96. [Crossref]

4. Gertow K, Wolbank S, Rozell B, Sugars R, Andang M, et al. (2004) Organized development from human embryonic stem cells after injection into immunodeficient mice. Stem Cells Dev 13: 421-435.

5. Ozolek JA, Castro CA (2011) Teratomas derived from embryonic stem cells as models for embryonic development, disease, and tumorigenesis. In: Kallos MS, editor. embryonic stem cells - basic biology to bioengineering. InTech 231-62.

6. Lensch MW, Schlaeger TM, Zon LI, Daley GQ (2007) Teratoma formation assays with human embryonic stem cells: a rationale for one type of human-animal chimera. Cell Stem Cell 1: 253-258. [Crossref]

7. Ramirez JM, Gerbal-Chaloin S, Milhavet O, Qiang B, Becker F, et al. (2011) Brief report: benchmarking human pluripotent stem cell markers during differentiation into the three germ layers unveils a striking heterogeneity: all markers are not equal. Stem Cells 29: 1469-1474.

8. Ramirez JM, Bai Q, Pequignot M, Becker F, Kassambara A, et al. (2013) Side scatter intensity is highly heterogeneous in undifferentiated pluripotent stem cells and predicts clonogenic self-renewal. Stem Cells Dev 22: 1851-1860.

9. Prokhorova TA, Harkness LM, Frandsen U, Ditzel N, Schroder HD, et al. (2009) Teratoma formation by human embryonic stem cells is site dependent and enhanced by the presence of Matrigel. Stem Cells Dev 18: 47-54. [Crossref]

10. Moll R, Divo M, Langbein L (2008) The human keratins: biology and pathology. Histochem Cell Biol 129: 705-733.
11. Botta MC, Ambu R, Liguori C, Van Eyken P, Pisanu A, et al. (2001) [CK20 expression in the gastrointestinal tract of the embryo and fetus]. Pathologica 93: 640-644. [Crossref]

12. Werling RW, Yaziji H, Bacchi CE, Gown AM (2003) CDX2, a highly sensitive and specific marker of adenocarcinomas of intestinal origin: an immunohistochemical survey of 476 primary and metastatic carcinomas. Am J Surg Pathol 27: 303-310.

13. Tot $\mathrm{T}$ (2002) Cytokeratins 20 and 7 as biomarkers: usefulness in discriminating primary from metastatic adenocarcinoma. Eur J Cancer 38: 758-763.

14. Dye BR, Hill DR, Ferguson MA, Tsai YH, Nagy MS, et al. (2015) In vitro generation of human pluripotent stem cell derived lung organoids. Elife 4.

15. Hawkins F, Kramer P, Jacob A, Driver I, Thomas DC, et al. (2017) Prospective isolation of NKX2-1-expressing human lung progenitors derived from pluripotent stem cells. J Clin Invest 127: 2277-2294.

16. Zorn AM, Wells JM (2009) Vertebrate endoderm development and organ formation. Annu Rev Cell Dev Biol 25: 221-251.

17. Damjanov I, Ahrlund-Richter L (2013) Characterization of Human Embryonic Stem Cell-derived Teratomas. In: Loring JF, Wesselschmidt RL, Schwartz PH, editors. Human Stem Cell Manuel 3:171-182.

18. Green MD, Chen A, Nostro MC, d'Souza SL, Schaniel C, et al. (2011) Generation of anterior foregut endoderm from human embryonic and induced pluripotent stem cells. Nat Biotechnol 29: 267-272. [Crossref]

19. Mou H, Zhao R, Sherwood R, Ahfeldt T, Lapey A, et al. (2012) Generation of multipotent lung and airway progenitors from mouse ESCs and patient-specific cystic fibrosis iPSCs. Cell Stem Cell 10: 385-397.

20. Huang SX, Islam MN, O'Neill J, Hu Z, Yang YG, et al. (2014) Efficient generation of lung and airway epithelial cells from human pluripotent stem cells. Nat Biotechnol 32: 84-91. [Crossref]

Copyright: (C2018 Sansac C. This is an open-access article distributed under the terms of the Creative Commons Attribution License, which permits unrestricted use, distribution, and reproduction in any medium, provided the original author and source are credited. 